\title{
Article
}

\section{Is there a link between self-perceived stress and physical activity levels in Scottish adolescents?}

Cowley, J., Kiely, J., and Collins, D.

Available at http://clok.uclan.ac.uk/18009/

Cowley, J., Kiely, J., ORCID: 0000-0001-9817-0224 and Collins, D. ORCID: 00000002-7601-0454 (2017) Is there a link between self-perceived stress and physical activity levels in Scottish adolescents? International Journal of Adolescent Medicine and Health . ISSN 0334-0139

It is advisable to refer to the publisher's version if you intend to cite from the work.

http://dx.doi.org/10.1515/ijamh-2016-0104

For more information about UCLan's research in this area go to http://www.uclan.ac.uk/researchgroups/ and search for <name of research Group>.

For information about Research generally at UCLan please go to http://www.uclan.ac.uk/research/

All outputs in CLoK are protected by Intellectual Property Rights law, including Copyright law. Copyright, IPR and Moral Rights for the works on this site are retained by the individual authors and/or other copyright owners. Terms and conditions for use of this material are defined in the policies page.

\section{CLoK}

Central Lancashire online Knowledge www.clok.uclan.ac.uk

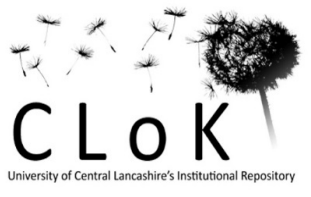


Is there a link between self-perceived stress and Physical Activity levels in

Scottish Adolescents? 0

Is there a link between self-perceived stress and Physical Activity levels in Scottish Adolescents?

\section{Joe Cowley ${ }^{\text {a }} *$ John Kiely ${ }^{\mathrm{a}}$ and Dave Collins ${ }^{\mathrm{a}}$}

a Institute of Coaching and Performance, School of Health and Wellness, University of Central Lancashire, Preston, PR1 2HE, United Kingdom, 
Is there a link between self-perceived stress and Physical Activity levels in

Scottish Adolescents? 1

\begin{abstract}
Purpose

It is becoming more evident that Physical Activity (PA) has a moderating effect on the negative health consequences of excessive psycho-social stress (Stults-Kolehmainen \& Sinha, 2014).

Recent literature has highlighted that the relationship between stress and physical activity is bidirectional (Stults-Kolehmainen \& Sinha, 2014). Furthermore, it has been suggested that the stress response impedes levels of participation in PA (Lutz, Stults-Kolehmainen, $\&$ Bartholomew, 2010). However, the impacts of accumulating life stress on PA remain under-investigated.
Aims:
i. To determine if significant differences exist in uptake of PA between adolescents who have experienced high life stress, in comparison to those who have not
ii. To investigate the relationship between perceived stress in adolescents and PA.
iii. To investigate whether high life stress can explain differences in other health behaviours, such as smoking and alcohol consumption

\title{
Methods
}

A purposive sampling strategy was employed. Adolescents from a low SES background who had experienced extensive life stress, were compared with more economicallyaffluent matched-controls. PA patterns were measured using the 'Physical Activity Questionnaire for High School (PAQA)'(Kowalski, Crocker, \& Kowalski, 1997) Stress scores were assessed using the 10 item version of the perceived stress scale (PSS-10). Statistical analysis was conducted

\section{Findings}

PA scores were significantly different between groups $(\mathrm{p}<0.05)$, with the low SES group significantly less active every day $(\mathrm{p}<0.05)$ except on Saturdays (Mann-Whitney $\mathrm{U}=31.0$, $\mathrm{Z}=-1.594, \mathrm{p}=>0.05)$. Furthermore, Spearman's correlation showed a negative relationship between total stress levels and PA during spare time $(r s=-0.61, n=10, p=$ $<0.05$ ). A similar relationship was evident for: PA levels during lunchtime, ( $r s=-0.69, n=$ $10, \mathrm{p}=<0.05$ ), evenings ( $\mathrm{rs}=-0.57, \mathrm{n}=10, \mathrm{p}=<0.05$ ) and for overall PA over a seven day period $(\mathrm{rs}=0.81, \mathrm{n}=10, \mathrm{p}=<0.05)$.

\section{Conclusion}

These findings add to existing evidence suggesting stress, during adolescent transition periods, impedes PA uptake. Physical Educators should incorporate the stress remediating effects of PA into school practice, and strive to inculcate leisure-based physical activities promoting sustainable PA, especially with adolescents likely to have been exposed to excessive stress loads during critical developmental periods. 
Is there a link between self-perceived stress and Physical Activity levels in Scottish Adolescents? 2

\section{The bidirectional effect of Physical Activity and stress}

The benefits of Physical Activity (PA) and its effect on associated health outcomes are strong and well documented (Cooper \& Hancock, 2011). Despite the overwhelming evidence of the benefits of PA and exercise, however, adherence to these behaviours remains problematic. For example, the Scottish Health survey in 2012 indicated that only $39 \%$ of adults (45\% of men, $33 \%$ of women) met the PA guidelines of 30 minutes of moderate activity on five or more days per week. Furthermore, since 2008 there had been no significant changes in the proportion of adults meeting these recommendations (The Scottish Government, 2012).

The PA practice of the Scottish population is of even greater importance, when considering reported health inequalities. The 'Glasgow effect', for example, is the term relating to specifically elevated levels of mortality in the Glasgow area, which when compared to other UK cities with similar levels of deprivation, remain unexplained (McCartney, Collins, Walsh, \& Batty, 2011). Accordingly, the need for research into the underpinning causes of low levels of PA seems pressing, especially when considering that $60 \%$ of young people will not participate in PA post compulsory education: a phenomenon labelled the post 16 gap (National Institute for Health and Clinical Excellence 2007).

Increasingly, current mental health concerns illustrate that additional PA benefits are relevant, as it is widely recognised that exercise has a positive effect on mental health outcomes (Bradford, 2016; Hamer, Stamatakis, \& Steptoe, 2009; Lutz et al., 2010). Perceived levels of wellbeing are not only greater in the physically active, perceived stress levels are also lower, thereby perpetuating positive spirals of improvement. In association, recent evidence highlights 
Is there a link between self-perceived stress and Physical Activity levels in

that childhood stress in 5-10 year olds is positively related to increases in BMI and waist-to-height ratio's. Subsequently highlighting the additive negative impacts of multiple poor lifestyle behaviours, such as low sleep duration, increased electronic screen time, consumption of high calorie snacks and accumulating life stress, on increased fat storage children,(Vanaelst et al., 2014). Further evidence is provided by a longitudinal study, conducted on 312 children aged 5-12, which reported that stress stimulated calorie intake, in the absence of hunger, thereby expediting weight gain. Additionally PA, as measured by accelerometers, increased in those with higher stress levels, suggesting that PA increased as a coping mechanism (Nathalie Michels et al., 2015). In contrast, quality of life, diet and PA measures, of 291 Swiss children (aged 3-6), illustrated that stress decreased PA levels, whilst simultaneously increasing consumption of high fat foods (N. Michels, Susi, Marques-Vidal, Nydegger, \& Puder, 2016). Certainly, the extant literature suggests excessive life stress impedes participation in PA (Lutz et al., 2010). Furthermore, the relationship between stress and PA are suggested to be bidirectional (Stults-Kolehmainen \& Sinha, 2014): regular PA reduces the negative consequences of stress, and less stressed people tend to more regularly participate in PA.

Despite these described benefits, however, evidence demonstrating the influence of stress on lifestyle behaviours and their link to obesity and physical health remains limited. The bulk of this evidence focusses on the positive effects of PA in blunting excessive stress reactivity (Stults-Kolehmainen \& Sinha, 2014). In this regard, Salmon (2001) suggests that exercise may produce neurochemical changes reducing depression and anxiety, thereby moderating reactivity to future 
Is there a link between self-perceived stress and Physical Activity levels in Scottish Adolescents? 4

stress (Salmon 2001). In addition, one recent review has highlighted evidence suggesting that experiencing stress may result in the individual becoming less physically active in the future (Stults-Kolehmainen \& Sinha, 2014).

The bidirectional nature of stress when associated with PA and, ultimately, its positive effect on health outcomes, places PA firmly in the frontline of the fight against the spectrum of ailments related to physical inactivity. Nevertheless, there remains a paucity of evidence investigating the effects of stress on the uptake of PA (Holmes, Ekkekakis, \& Eisenmann, 2010; Stults-Kolehmainen \& Sinha, 2014).

\section{Increasing PA in Adolescents}

Adolescence is recognised as a critical time for instilling positive health behaviours such as PA (Pearson, Atkin, Biddle, Gorely, \& Edwardson, 2009). Additionally, increasing PA levels in adolescents has been shown to reduce somatic complaints in a European sample (Ferron, Narring, Cauderay, \& Michaud, 1999). In contrast, the Swiss adolescent health survey reported that adolescents with lower levels of sports- based activities were more likely to adopt negative experimental behaviours, such as smoking cigarettes and marijuana, and increased risk taking behaviours, such as not wearing a car seat belt (Ferron, Narring, Cauderay, \& Michaud, 1999). This survey suggested PA as a potent component of any comprehensive strategy aimed at reducing negative health behaviours among adolescents.

Additionally, further evidence suggests that active young people, deemed as athletic, are better inclined to maintain health status at a satisfying level and are better prepared to cope with potential health problems (Ferron, Narring, Cauderay, 
Is there a link between self-perceived stress and Physical Activity levels in Scottish Adolescents? 5

\& Michaud, 1999) In fact, even small amounts of structured activity outside of school hours has demonstrated positive improvements in the emotional well-being of adolescents

\section{The importance of life changing events and changes in PA}

Lifestyle upheaval, such as changing or leaving school or employment, may significantly influence an individual's daily routine and elevate background levels of psychosocial stress (Engberg, 2010): thereby exerting a negative impact on PA levels in adulthood (Allender, Cowburn, \& Foster, 2006). Changes in employment status, residence, perceived physical appearance, relationships and in family structure are all common life events serving to raise background levels of psychosocial stress in adolescent age groups (Allender et al., 2006; Allender, Hutchinson, \& Foster, 2008).

There is good evidence illustrating the impact of these transitions. Systematic reviews of qualitative and quantitative research investigating barriers and motivations to PA report that many life changing events exerted a negative effect on participation in PA levels with the most common changes experienced at the transition phase from adolescence to adulthood, the time when the cessation of compulsory education approaches (Allender et al., 2006; Allender et al., 2008). A period of life which, as already noted, is a critical time for establishing lifelong health habits. Indeed, the literature has illustrated that a shift in life course, particularly between ages $15-16$, has implications for participation in future PA.

Notably, the overall ideology behind the Curriculum for Excellence in Scotland is to optimise lifelong health, wellbeing and create opportunities for lifelong PA and learning (Niven, Fawkner, Knowles, Henretty, \& Stephenson, 2009; The Scottish Government, 2009). To date, the extant literature highlights 
Is there a link between self-perceived stress and Physical Activity levels in Scottish Adolescents? 6

the need for emphasising the importance of quality PE with less emphasis on traditional games based drills and more on sustainable, enjoyable activity to ensure the maintenance of PA into adulthood.(Fairclough, Stratton, \& Baldwin, 2002; Kirk, 2005). Furthermore, the need for pupil input and student voice is seen as critical in order to promote sustainable PE and PA.

The move away from controlling, temporary forms of motivation can be supported by a culture where teachers listen to and then act upon what pupils have to say about their physical education experiences, alternative provision that has the capacity to promote autonomous, more enduring forms of motivation in those female and male pupils who do not enjoy games (Forsyth, 2014).

Finally, while previous authors have identified the importance of stress and the effect this has on health behaviours but the failure to identify specific dimensions of stress and the paucity of evidence focussing on a narrower focus on parameters such as PA has deemed it necessary to investigate the effects stress may have on behaviours such as PA (Pampel, Krueger, \& Denney, 2010).

\section{Research aims}

In order to add to this growing body of knowledge, this paper focussed on identifying if there were significant differences in PA participation between adolescents who had experienced high life stress, in comparison to those who had not.

\section{Method}

\section{Participants}

A purposive sampling strategy was employed, with participants recruited on the basis of socioeconomic factors and characteristics using the Scottish Index of Multiple Deprivation (SIMD) as guidance. Twenty participants (mean age 16.1, $S D \pm .31)$ were recruited for this study. Group one consisted of10 individuals from 
Is there a link between self-perceived stress and Physical Activity levels in

the PA3, G32 and G40 postcodes in Glasgow; an underprivileged area with low income and poor health outcomes. Group 2 consisted of 10 participants matched on age and sex, drawn from the postcodes FK1, G67 and G68 a background that was not deemed to be underprivileged.

\section{Instrumentation}

The study used validated questionnaires to measure self-perceived stress and PA, then subsequently analysed the relationship that may exist between stress and health behaviours such as PA, smoking, poor diet and substance misuse. The validated questionnaire 'Perceived Stress Scale for Adolescents (Cohen et al.1988) was used for data collection, as this instrument has been shown to be a useful measure for detecting the relationship between stress and other health related measures. PA patterns were measured using the modified version of the questionnaire Physical Activity Questionnaire for High School (PAQA (Kowalski et al., 1997) which is a seven day self -administered recall questionnaire deemed valid and reliable for adolescents of 14-17 years of age. Other health behaviours such as smoking and substance misuse were collected using both quantitative and an open qualitative comments box in the questionnaire.

\section{Procedure}

Ethics was sought and received from the University of Central Lancashire Ethics Committee. Informed consent was sought and a briefing sheet provided for all participants informing them of the nature of the study and the option to opt out at any time. All provided informed consent, with counter signatures provided by their parent or guardian where necessary. Data were collected through use of the various instruments, administered personally by the first author, and then analysed using SPSS v20. Independent sample t tests were used when the data followed a 
Is there a link between self-perceived stress and Physical Activity levels in Scottish Adolescents? 8

normal distribution. This included age and total stress scores. Non parametric tests in the form of Mann-Whitney $U$ test were used when the data were skewed, to analyse the difference between the two independent groups. Additionally, Spearman's rank correlation was carried out to analyse the strength of the correlation between total PA scores and total stress scores for each group respectively.

\section{Results}

The first step was to ensure the appropriate equivalence and differences between the two sample groups. There was no significant difference in age ( $p>.05)$. In contrast, a significant difference was detected in total stress score against a possible ceiling of 40 with Group 1 showing a total of $34.3(S D \pm 4.72)$, whilst Group 2 displayed a total of $18.6(S D \pm 3.94, p<0.05)$. 
Is there a link between self-perceived stress and Physical Activity levels in Scottish Adolescents? 9

Table 1 below shows Mann- Whitney values for PA scores, level of smoking and drinking over a seven days period.

$\underline{\text { Table } 1}$

\begin{tabular}{|c|c|c|c|c|c|}
\hline PA on: & $\begin{array}{l}\text { Mann } \\
\text { Whitney }\end{array}$ & $\begin{array}{l}\text { Wilcoxon } \\
\text { W }\end{array}$ & Z Value & $\begin{array}{l}\text { Asymp. Sig. } \\
\text { ( } 2 \text { tailed) }\end{array}$ & $\begin{array}{l}\text { Exact } \\
\text { Sig. }\end{array}$ \\
\hline Mon & 7.000 & 62.000 & -3.401 & 0.001 & $0.000 *$ \\
\hline Tue & 0.000 & 55.000 & -3.907 & 0.000 & $0.000^{*}$ \\
\hline Wed & 3.000 & 58.000 & -3.821 & 0.000 & $0.000 *$ \\
\hline Thu & 17.500 & 72.500 & -2.784 & 0.005 & $0.011^{*}$ \\
\hline Fri & 18.000 & 73.000 & -2.749 & 0.006 & $0.015^{*}$ \\
\hline Sat & 31.000 & 86.000 & -1.594 & 0.111 & 0.165 \\
\hline Sun & 12.500 & 67.500 & -2.999 & 0.003 & $0.003 *$ \\
\hline Smoke & 15.000 & 70.000 & -3.067 & 0.002 & $0.007^{*}$ \\
\hline Drink & 30.000 & 85.000 & -1.780 & 0.075 & 0.143 \\
\hline
\end{tabular}


Is there a link between self-perceived stress and Physical Activity levels in Scottish Adolescents? 10

Next, we checked for between group differences in PA, smoking and drinking; these data are presented in Table 1. As shown, total PA scores were significantly different $(\mathrm{p}<0.05)$, with the low SES group significantly less active on every day except for Saturday.-The low SES adolescent group also showed a significantly higher number of smokers in comparison to the non SES group. However, as shown in Table 1 there was no significant difference in the levels of drinking evident between groups.

To examine relationships between stress and PA across individuals, Spearman's correlation was conducted to determine the relationship between the total stress score and PA during spare time, during PE, immediately after school, evening's weekends and total PA levels perception. In the low SES group, there was a strong negative relationship between total stress levels and PA during spare time $\left(r_{s}=-0.61, p<0.05\right)$. A similar relationship was evident for: PA levels during lunchtime, $\left(r_{s}=-0.69, p<0.05\right)$, during evenings $\left(r_{s}=-0.57, p<0.05\right)$ and for overall PA over a seven day period $\left(\mathrm{r}_{\mathrm{s}}=0.81, \mathrm{p}<0.05\right)$. However, no relationship was evident between reported participation levels in PE and total stress scores $\left(r_{s}=0.30, p=>0.05\right)$.

In contrast, no significant relationships between total stress level and PA were evident in the control group, across all variables $(\mathrm{p}=>0.05)$. 
Is there a link between self-perceived stress and Physical Activity levels in Scottish Adolescents? 11

\section{Discussion}

The aim of this study was to determine if there was a difference in PA levels between those that had experienced greater biopsychosocial life stress as compared with those that have not. The acknowledgement that greater stress levels have an effect on PA levels and structured exercise programmes in all but long term exercisers is of particular interest to us, as we believe that stress may play a synergistic role in the lives of those individuals typified by the Glasgow effect (Lutz et al., 2010).

Whilst it is regularly acknowledged that PA plays a role in repelling the negative effects of stress, there is a lack of evidence as to how this stress affects the levels of PA. The findings from this study illustrate a clear diminishment of PA levels in those young people who have experienced, or are experiencing, elevated levels of perceived stress.

This evidence suggests a negative relationship between high total stress scores and levels of PA. This relationship was statistically significant for PA recall during lunch periods, immediately after school and over a seven day period. Whilst methodological limitations are evident in PA recall, this study adds to the limited body of existing evidence supporting bidirectional interplay between of PA and stress (Holmes et al., 2010; Lutz et al., 2010; Stults-Kolehmainen \& Sinha, 2014). Furthermore, the findings confirm the effects of excess stress on health behaviours, such as PA during transitional phases of life (Allender et al., 2006; Allender et al., 2008)

Interestingly, whilst the results highlight a significant difference between groups for participation during PE classes, with the control group significantly 
Is there a link between self-perceived stress and Physical Activity levels in Scottish Adolescents? 12

more active than the low SES group, there is no significant association, either positive or negative, to suggest that Physical Education class participation is affected by those who scored either high or low in the administered questionnaire. This may highlight the effectiveness of compulsory Physical Education in ensuring that short-term PA guidelines are met. More concerning, however, although data suggest that those with higher stress scores are likely to reach their peak levels of PA during PE lessons, traditional methods of teaching physical education may be ineffective in promoting lifelong PA, typically exhibiting a very weak transfer of learning from school PE to adult life (Casey \& Goodyear, 2015), This circumstance may be attributable to PE's traditional overreliance on sport based drills to improve skill, with less emphasis on fun, enjoyment, leisure and inclusion. Accordingly it has been suggested that a more updated holistic model based approach is preferable to ensure effective promotion of lifelong PA (Casey \& Goodyear, 2015; Fairclough et al., 2002; Kirk, 2013).

These data also highlight the importance of continuing PA beyond the school aged years into adult life. Previous authors have identified that PE should be seen as a vehicle to educate pupils on the need to sustain PA throughout life. A position suggesting that a special emphasis on recreational and leisure based content might be more beneficial over the life-span, than standard sports-based PA that is conventionally seen as a 'must do', or compulsory element of the curriculum (Kirk, 2005, 2013). In this regard, Fairclough, Stratton and Baldwin (2002) emphasise that physical educators must recognise which activities have the greatest potential for 'carry-over' value into adult life (Fairclough et al., 2002). Additionally, the implementation of PE models which focus on developing skills at the critical early stages of development are imperative(Giblin, Collins, 
Is there a link between self-perceived stress and Physical Activity levels in Scottish Adolescents? 13

MacNamara, \& Kiely, 2014; MacNamara, Collins, \& Giblin, 2015). There is also a real need for research to examine which aspects of PE, and when in the child's life, are most effective at promoting lifelong PA (MacNamara et al., 2015).

\section{Drinking and smoking}

It has been established that, for those from areas of lower socio-economic status, the behaviours of smoking and drinking are more pronounced, with heavier patterns of use observed. Of course, it must be acknowledged that poor dietary habits, alcohol, tobacco consumption and recreational drug-use habits are widespread across all social classes. Our results showed a significant difference in those who smoke cigarettes but not in alcohol consumption, with both groups showing similar patterns. This may be down to the low sample size or, perhaps more likely, to be down to the 'acceptability' of alcohol in youngsters' lives today (Seaman \& Ikegwuonu, 2010).

\section{Limitations}

Results notwithstanding, the limitations of the study should be acknowledged. One limitation relates to the low sample size, however as a significant association was found it is important to clarify that a larger confirmatory study may be needed to strengthen these findings. Nevertheless, this was out with the allocatable resources of this project. PA measurement using questionnaires may result in recall bias, as participants may find it difficult to accurately evaluate their activity patterns on a particular week. Furthermore, research questionnaires such as the PAAQ-A may not accurately evaluate the intensity of the PA. Notably, the literature validating the questionnaire used in this study have supported its use for assessing general PA levels for the purpose seen 
Is there a link between self-perceived stress and Physical Activity levels in Scottish Adolescents? 14

in this study as a reliable estimate of PA levels, (Kowalski, Crocker, \& Faulkner, 2004). Additionally a modified version of the questionnaire has been previously used on a large cohort of primary and secondary aged school children in Scottish schools, with emphasis on this transitional period (Inchley, Kirby, \& Currie, 2008).

\section{Conclusions and implications for future practice}

The main objectives of this study were to determine if there is a significant difference in uptake of PA between adolescents who have experienced high life stress, in comparison to those who have not. Whilst also considering the role that the Physical Education curriculum may play in facilitating uptake when considering stressful outcomes. PA levels were shown to significantly differ in those adolescents who displayed high stress scores when compared their peers who had been subjected to less accumulative life stress. The findings presented here, add to the evidence-base illustrating that prior stress impedes PA uptake during transitional phases of life.

This study placed special emphasis on the developing adolescent and how life stressors can negatively influence health behaviours, which may eventually lead to negative health outcomes. Effective uptake of PA is reliant, not only upon participation in PA through Physical Education, but also on providing young people with a well-rounded sampling of PA experiences, beyond simply those considered as traditional PE-based games. Physical Educators should understand the stress remediating effects of PA, and strive to incorporate more activities that have a 'carry over value' in to adulthood in order to promote sustainable PA. 
Is there a link between self-perceived stress and Physical Activity levels in Scottish Adolescents? 15

Furthermore, those involved in designing the curriculum should strive to provide a positive, inclusive experience for everyone with the emphasis on 'a task climate', rather than an overemphasis on determined 'winning' (Kirk, 2005). Recent trends in research tend to focus on the amount of time spent being physically active in classes or at that time of life. More importantly, however, there should be a structured curriculum content prioritising sustainable PA for life, rather than placing sole emphasis on the current level of PA in the young participant (Casey \& Goodyear, 2015; Green, 2004).

\section{References}

Allender, Steven, Cowburn, Gill, \& Foster, Charlie. (2006). Understanding participation in sport and physical activity among children and adults: a review of qualitative studies. Health Education Research, 21(6), 826-835. doi: 10.1093/her/cyl063

Allender, Steven, Hutchinson, Lauren, \& Foster, Charles. (2008). Life-change events and participation in physical activity: a systematic review. Health Promotion International, 23(2), 160-172. doi: 10.1093/heapro/dan012

Bradford, E. (2016). In the Mind: Key facts about mental health in Scotland. from http://www.bbc.co.uk/news/uk-scotland-35576995

Casey, Ashley, \& Goodyear, Victoria A. (2015). Can Cooperative Learning Achieve the Four Learning Outcomes of Physical Education? A Review of Literature. Quest, 67(1), 56-72. doi: 10.1080/00336297.2014.984733

Cooper, K, \& Hancock, C. (2011). Review: The benefits of physical activity for health and well-being C3 Collaborating for Health*.

Engberg, Elina. (2010). Life Change evebts and physical activity:A Systematic Review. (MSc), University of Eastern Finland, Finland.

Fairclough, Stuart, Stratton, Gareth, \& Baldwin, Graham. (2002). The Contribution of Secondary School Physical Education to Lifetime Physical Activity. European Physical Education Review, 8(1), 69-84. doi: 10.1177/1356336x020081005

Ferron, C., Narring, F., Cauderay, M., \& Michaud, P.-A. (1999). Sport activity in adolescence: associations with health perceptions and experimental behaviours. Health Education Research, 14(2), 225-233. doi: 10.1093/her/14.2.225

Forsyth, S. (2014). CfE and the Donaldson Report: A coherent approach supporting teachers in the move towards evidence based practice. SATPE Biannual E-Journal - Nov 2014 Edition, 1(2).

Giblin, Susan, Collins, David, MacNamara, Aine, \& Kiely, John. (2014). "Deliberate Preparation" as an Evidence-Based Focus for Primary Physical Education. Quest, 66(4), 385-395. doi: 10.1080/00336297.2014.944716 
Is there a link between self-perceived stress and Physical Activity levels in Scottish Adolescents? 16

Green, Ken. (2004). Physical education, lifelong participation and 'the couch potato society'1. Physical Education and Sport Pedagogy, 9(1), 73-86. doi: $10.1080 / 1740898042000208133$

Hamer, M, Stamatakis, E, \& Steptoe, A. (2009). Dose-response relationship between physical activity and mental health: the Scottish Health Survey. British Journal of Sports Medicine, 43(14), 1111-1114. doi: 10.1136/bjsm.2008.046243

Holmes, M. E., Ekkekakis, P., \& Eisenmann, J. C. (2010). The physical activity, stress and metabolic syndrome triangle: a guide to unfamiliar territory for the obesity researcher. Obesity reviews : an official journal of the International Association for the Study of Obesity, 11(7), 492-507.

Inchley, J, Kirby, J , \& Currie, C. (2008). Physical activity among adolescents in Scotland Final Report of the PASS Study. Edinburgh.

Kirk, David. (2005). Physical education, youth sport and lifelong participation: the importance of early learning experiences. European Physical Education Review, 11(3), 239-255. doi: 10.1177/1356336x05056649

Kirk, David. (2013). Educational Value and Models-Based Practice in Physical Education. Educational Philosophy and Theory, 45(9), 973-986. doi: 10.1080/00131857.2013.785352

Kowalski, K. C., Crocker, P. R. E., \& Faulkner, R. A. (2004). The Physical Activity Questionnaire for Older Children $(P A Q-C)$ and Adolescents (PAQ-A) Manual British Journal of Sports Medicine

Kowalski, K. C., Crocker, P. R. E., \& Kowalski, N. P. (1997). Convergent Validity of the Physical Acivity Questionnaire for Adolescents.

. Pediatric Exercise Science, 9, 342-352.

Lutz, Rafer S., Stults-Kolehmainen, Matthew A., \& Bartholomew, John B. (2010). Exercise caution when stressed: Stages of change and the stress-exercise participation relationship. Psychology of Sport and Exercise, 11(6), 560567. doi: http://dx.doi.org/10.1016/j.psychsport.2010.06.005

MacNamara, Áine, Collins, Dave, \& Giblin, Susan. (2015). Just let them play? Deliberate preparation as the most appropriate foundation for lifelong physical activity. Frontiers in Psychology, 6, 1548. doi: 10.3389/fpsyg.2015.01548

McCartney, G, Collins, C, Walsh, D , \& Batty, D. (2011). Accounting For Scotland's Excess Mortality:Towards a Synthesis (Hypothesis Report): Glasgow Centre for Population Health (GCPH).

Michels, N., Susi, K., Marques-Vidal, P. M., Nydegger, A., \& Puder, J. J. (2016). Psychosocial Quality-of-Life, Lifestyle and Adiposity: A Longitudinal Study in Pre-schoolers (Ballabeina Study). Int J Behav Med, 23(3), 383392. doi: 10.1007/s12529-016-9537-z

Michels, Nathalie, Sioen, Isabelle, Boone, Liesbet, Braet, Caroline, Vanaelst, Barbara, Huybrechts, Inge, \& De Henauw, Stefaan. (2015). Longitudinal association between child stress and lifestyle. Health Psychology, 34(1), 40.

Niven, Ailsa, Fawkner, Samantha, Knowles, Ann-Marie, Henretty, Joan, \& Stephenson, Claire. (2009). Social physique anxiety and physical activity in early adolescent girls: The influence of maturation and physical activity motives. Journal of Sports Sciences, 27(3), 299-305. doi: 10.1080/02640410802578164 
Is there a link between self-perceived stress and Physical Activity levels in Scottish Adolescents? 17

Pampel, Fred C., Krueger, Patrick M., \& Denney, Justin T. (2010). Socioeconomic Disparities in Health Behaviors. Annual Review of Sociology, 36(1), 349370. doi: doi:10.1146/annurev.soc.012809.102529

Pearson, Natalie, Atkin, Andrew, Biddle, Stuart, Gorely, Trish, \& Edwardson, Charlotte. (2009). Patterns of adolescent physical activity and dietary behaviours. International Journal of Behavioral Nutrition and Physical Activity, 6(1), 45.

Seaman, P , \& Ikegwuonu, T. (2010). Drinking to belong.Understanding young adults' alcohol use within social networks (pp. 1-55). Glasgow: Glasgow Centre for Public Health and the Joseph Rowntree Foundation.

Stults-Kolehmainen, MatthewA, \& Sinha, Rajita. (2014). The Effects of Stress on Physical Activity and Exercise. Sports Medicine, 44(1), 81-121. doi: 10.1007/s40279-013-0090-5

The Scottish Government. (2009). Curriculum for excellence building the curriculum 4:skills for learning, skills for learning, skills for life and skills for work. Edinburgh: Retrieved from http://www.gov.scot/resource/doc/288517/0088239.pdf.

The Scottish Government. (2012). Physical Activity levels based on the Scottish Health Survey. Retrieved 27/01/16, 2016

Vanaelst, Barbara, Michels, Nathalie, Clays, Els, Herrmann, Diana, Huybrechts, Inge, Sioen, Isabelle, . . . De Henauw, Stefaan. (2014). The Association Between Childhood Stress and Body Composition, and the Role of StressRelated Lifestyle Factors-Cross-sectional Findings from the Baseline ChiBS Survey. International Journal of Behavioral Medicine, 21(2), 292301. doi: 10.1007/s12529-013-9294-1 